\title{
The efficacy of microperimetry and contrast sensitivity test in the diagnosis of optic chiasm compression due to pituitary adenomas
}

\author{
Monika Sarnat-Kucharczyk', Beata Kos-Kudła', ${ }^{2}$, Dariusz Kajdaniuk³, Ewa Mrukwa-Kominek1 \\ ${ }^{1}$ Department of Ophthalmology, School of Medicine in Katowice, Medical University of Silesia, Katowice, Poland \\ ${ }^{2}$ Department of Endocrinology and Neuroendocrine Tumors, Medical University of Silesia, Katowice, Poland \\ ${ }^{3}$ Department of Pathophysiology and Endocrinology, Medical University of Silesia, Katowice, Poland
}

\begin{abstract}
Introduction: The aim of the study was to determine which microperimetry and contrast sensitivity test parameters would prove the most valuable during diagnosing optic chiasm compression due to pituitary adenomas.

Material and methods: A control group comprised healthy individuals (Group 1). Patients with pituitary macroadenoma were divided into two groups: Group 2 - absent optic chiasm compression; and Group 3 - present optic chiasm compression detected on contrastenhanced magnetic resonance imaging (MRI). Each group comprised 20 patients (40 eyes), i.e. a total of 60 patients (120 eyes) were examined. A complete ocular examination, intraocular pressure, microperimetry, contrast sensitivity test, kinetic Goldmann visual field, and static Octopus visual field test were performed.

Results: Group 1 and 2 variables showed no statistically significant differences with respect to the mean sensitivity (MS) and mean defect (MD) in microperimetry. After dividing the microperimetry area into quadrants, a difference was shown in the mean sensitivity of the lower-nasal quadrant (MS LN) and mean defect of the lower-nasal quadrant (MD LN) between those groups. Receiver operating characteristic (ROC) curves analysis revealed that the microperimetry parameter - MS LN as well as row D and E contrast sensitivity test could be highly specific in the assessment of early damage of the optic nerve in patients suffering from pituitary adenoma.

Conclusions: Microperimetry and contrast sensitivity test are non-invasive diagnostic investigations adjunctive to MRI, which facilitate detection of early chiasmal compression caused by pituitary adenomas. (Endokrynol Pol 2019; 70 (3): 241-247)

Key words: microperimetry; contrast sensitivity; pituitary adenoma; optic chiasm
\end{abstract}

\section{Introduction}

Pituitary adenomas are a heterogeneous group of tumours in terms of hormone secretion and growth characteristics [1,2]. With such heterogeneity of tumours, no obvious and clear treatment algorithm is present [3]. The majority of pituitary adenomas are sporadic, and only $5 \%$ of them occur in a familial setting [4].

Although some pituitary tumours remain clinically silent for a long time and may grow slowly for years, other tumours may present aggressive and rapid growth. These are usually macroadenomas with invasive growth, high recurrence rate, and resistance to repeated treatments $[5,6]$.

Microperimetry, also known as fundus perimetry, is characterised by significantly higher central retinal sensitivity compared to conventional static perimetry. It allows detection of the slightest changes in retinal sensitivity even when a fundus examination does not reveal any abnormalities [7]. Mean retinal sensitivity is a valuable complementary measurement to visual acuity.

Contrast sensitivity test is an important test for functional vision, including the Functional Acuity Contrast Test (FACT) developed by Arthur P. Ginsburg. In this examination the patient is asked to identify the orientation of lines in a grating pattern at various levels of contrast and spatial frequency.

Contrast-enhanced NMR is the gold standard in the diagnosis of adenomas and helps precisely define tumour boundaries and its relationship with adjacent structures, such as the optic chiasm [8]. High sensitivity allows detection of small tumours (microadenomas). When growing, macroadenomas extend into the suprasellar region and towards the optic chiasm, they may lift up third ventricle floor, obstruct the foramen 
of Monro, or extend sideways towards the cavernous sinus or downwards to the sphenoid sinus [9].

Kinetic visual field (VF) is very useful at advanced stages of the disease and in patients who have difficulties in performing a static VF. Although Octopus static perimetry test is helpful in the diagnosis of pituitary tumours, it may not reveal early optic nerve damage [10] and it should be noted that prolonged compression to the optic chiasm may produce deep and irreversible visual field defects [11].

The aim of the study was to determine which microperimetry and contrast sensitivity test parameters would prove the most valuable during diagnosing optic chiasm compression due to pituitary adenomas.

\section{Material and methods}

The study was consistent with the principles of the Declaration of Helsinki and was approved by the Ethics Committee of the Medical University of Silesia, Katowice, Poland. All the subjects gave informed consent to participate in the research.

Forty patients ( 80 eyes) with pituitary macroadenoma were enrolled in the study. The control group (Group 1) included 20 healthy patients - 11 women and nine men (40 eyes). Patients with macroadenoma were divided into two groups: Group 2 (11 women and 9 men) - absent optic chiasm compression, Group 3 (14 women and 6 men) - present optic chiasm compression detected on contract-enhanced NMR. A total of 60 patients (120 eyes) aged $46.48 \pm 12.47$ years were examined.

The inclusion criteria for study groups were as follows:

- informed consent to participate in the study;

- pituitary macroadenoma detected on contrast-enhanced NMR;

- normal intraocular pressure;

- transparency of the ocular media;

- no myopia or hyperopia $\geq 4$ dioptres, or astigmatism $\geq 2$ dioptres;

- no corneal, lens, or retinal abnormalities;

- no history of ocular surgery;

- no systemic diseases considered contraindications to microperimetry or contrast sensitivity test;

- no severe visual defects precluding microperimetry or contrast sensitivity test. The participants reported no addictions.

All subjects participating in the study underwent ophthalmic examination of both eyes, including near and distance bestcorrected visual acuity, slit lamp examination of the anterior and posterior segments, microperimetry (MP-1, Nidek Technologies, Italy, pattern: macula $12^{\circ} 10 \mathrm{~dB}$, stimulus: Goldmann III, $\left.200 \mathrm{~ms}\right)$, Functional Acuity Contrast Test (FACT, Optec 6500, Stereo Optical Co. Inc., Chicago, IL, USA), kinetic VF (Goldmann perimeter, Haag-Streit Inc., Bern, Switzerland, stimulus: 4 III), and static VF (Octopus 1-2-3 perimeter, Haag-Streit Inc., Koenic, Switzerland, strategy: TOP).

Division of the examined macular region into quadrants, not available in the MP- 1 software, enabled us to accurately assess the central retinal sensitivity of each quadrant (Fig. 1).

The aim of the study was to determine which microperimetry and contrast sensitivity test parameters would prove the most valuable during diagnosing chiasmal compression due to pituitary adenomas.

\section{Statistical analysis}

$P$ value $<0.05$ was determined as statistically significant. The Shapiro-Wilk test and Kolmogorov-Smirnov test were performed to test for normality. Differences between two groups were compared with the non-parametric Mann-Whitney $U$ test, while the Kruskal-

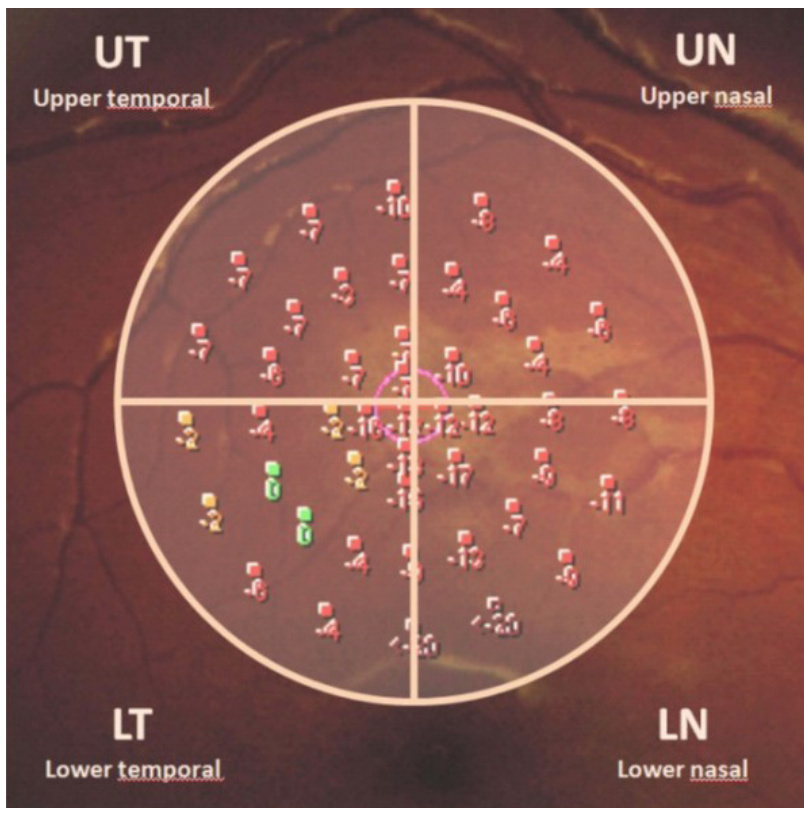

Figure 1. Division of the examined macular region by microperimetry into four quadrants

-Wallis test with post hoc Dunn's test was used to compare outcomes among more than two groups. The t-test was used to compare normal distributions, and, if needed, the Bonferroni post-hoc test. ROC analysis was applied to compare different variables used in the diagnosis of pituitary macroadenomas.

\section{Results}

A total of 120 eyes of 60 participants (mean age \pm SD: $46.48 \pm 12.47$ years, 24 males, 36 females) were included in the study. The groups did not differ significantly with respect to the participants' age. Tables I-III present the most important study variables in the control and study groups.

Best-corrected distance visual acuity was 1.0, 0.99, and 0.97 in Group 1, 2, and 3, respectively. Statistically significant differences were only noted between Group 1 and 3 ( $p=0.005351)$. Best-corrected near visual acuity was 0.5 in Group 1, 0.52 in Group 2, and 0.56 in Group 3; again, statistically significant differences were only seen between Group 1 and 3 ( $p=0.000848)$.

To the mean sensitivity of the macula by microperimetry was $18.91 \mathrm{~dB}, 18.3 \mathrm{~dB}$, and $13.39 \mathrm{~dB}$ in Group 1,2 , and 3, respectively (Fig. 2). The mean defect was: $-1.07 \mathrm{~dB}$ in Group 1, $-1.66 \mathrm{~dB}$ in Group 2, and $-5.1 \mathrm{~dB}$ in Group 3 (Fig. 3).

Group 1 and 2 did not differ significantly with respect to MS and MD by microperimetry. However, after dividing the microperimetry area into quadrants, a difference was demonstrated in MS LN and MD LN between those groups. Figure 4 depicts a comparison of MS LN in the study and control groups. 
Table I. Variables in Group 1

\begin{tabular}{lllllllc}
\hline Group 1 & & N & Mean & SD & Median & Min & Max \\
\hline \multirow{3}{*}{ Microperimetry [dB] } & MS & 40 & 18.91 & 0.60 & 18.90 & 17.80 & 20.00 \\
\cline { 2 - 8 } & MD & 40 & -1.07 & 0.57 & -1.10 & -2.20 & 0 \\
\cline { 2 - 8 } & MS LN & 40 & 18.96 & 0.68 & 18.93 & 17.45 & 20.00 \\
\hline \multirow{3}{*}{$\begin{array}{l}\text { Contrast sensitivity test } \\
\text { (patch scores) }\end{array}$} & Row A & 40 & 6.44 & 1.22 & 5.70 & 5.00 & 8.70 \\
\cline { 2 - 8 } & Row B & 40 & 6.67 & 1.21 & 6.70 & 4.00 & 8.70 \\
\cline { 2 - 8 } & Row C & 40 & 6.89 & 0.81 & 7.00 & 5.00 & 8.00 \\
\cline { 2 - 8 } & Row D & 40 & 6.03 & 1.01 & 6.00 & 4.00 & 8.00 \\
\cline { 2 - 8 } & Row E & 40 & 4.04 & 1.43 & 4.00 & 2.00 & 7.00 \\
\hline \multirow{3}{*}{ Static VF - 0ctopus [dB] } & MS & 40 & 28.36 & 1.34 & 28.25 & 24.30 & 30.10 \\
\cline { 2 - 8 } & MD & 40 & 0.15 & 0.99 & 0.10 & -1.70 & 3.00 \\
\hline & MS LN & 40 & 28.36 & 1.36 & 28.35 & 25.00 & 30.50 \\
\hline Kinetic VF - temporal part [degrees] & 40 & 83.50 & 4.83 & 85.00 & 70.00 & 90.00 \\
\hline
\end{tabular}

$\mathrm{N}$ — number; SD — standard deviation; Min — minimum; Max — maximum; dB - decibels; MS — mean sensitivity; MD — mean defect; MS LN — mean sensitivity lower-temporal, VF — visual field

Table II. Variables in Group 2

\begin{tabular}{|c|c|c|c|c|c|c|c|}
\hline Group 2 & & $\mathbf{N}$ & Mean & SD & Median & Min & Max \\
\hline \multirow{3}{*}{ Microperimetry [dB] } & MS & 40 & 18.3 & 0.94 & 18.15 & 16.20 & 19.50 \\
\hline & $\mathrm{MD}$ & 40 & -1.66 & 1.12 & -1.65 & -3.70 & 2.10 \\
\hline & MS LN & 40 & 17.49 & 1.04 & 17.40 & 15.63 & 19.38 \\
\hline \multirow{5}{*}{$\begin{array}{l}\text { Contrast sensitivity test } \\
\text { (patch scores) }\end{array}$} & Row $A$ & 40 & 4.80 & 1.36 & 5.00 & 1.70 & 8.30 \\
\hline & Row B & 40 & 4.98 & 1.67 & 5.00 & 1.00 & 8.00 \\
\hline & Row C & 40 & 5.00 & 1.85 & 5.00 & 0.30 & 8.70 \\
\hline & Row D & 40 & 3.25 & 1.66 & 3.15 & 0.30 & 7.00 \\
\hline & Row $\mathrm{E}$ & 40 & 1.50 & 1.24 & 1.00 & 0 & 5.00 \\
\hline \multirow{3}{*}{$\begin{array}{l}\text { Static VF } \\
- \text { Octopus [dB] }\end{array}$} & MS & 40 & 26.28 & 1.78 & 26.30 & 22.80 & 29.80 \\
\hline & $\mathrm{MD}$ & 40 & 2.18 & 2.09 & 2.10 & -1.70 & 7.00 \\
\hline & MS LN & 40 & 25.16 & 2.84 & 25.00 & 16.00 & 30.00 \\
\hline \multicolumn{2}{|c|}{ Kinetic VF — temporal part [degrees] } & 40 & 79.00 & 6.52 & 80.00 & 60.00 & 90.00 \\
\hline
\end{tabular}

$\mathrm{N}$ — number; SD — standard deviation; Min — minimum; Max — maximum; dB - decibels; MS — mean sensitivity; MD — mean defect; MS LN — mean sensitivity lower-temporal, VF — visual field

The average duration of the microperimetry test for one eye was $8 \mathrm{~min} 30 \mathrm{~s}$ in Group 1, 9 min $4 \mathrm{~s}$ in Group 2, and $10 \mathrm{~min} 56 \mathrm{~s}$ in Group 3. No statistically significant differences were noted between Group 1 and 2. Statistically significant differences were revealed between Group 1 and $3(p=0.000113)$ and Group 2 and $3(p=0.000120)$.

Group 1 participants had normal contrast sensitivity (rows A-E). In the remaining two groups a reduction was noted in all spatial frequencies with the lowest values obtained in Group 3. A comparison of contrast sensitivity between Group 1 and 2 as well as Group 1 and 3 revealed statistically significant differences at all spatial frequencies. An analysis of the same variables in Group 2 and 3 showed statistically significant differences but only in rows $A, C, D$.

An analysis of MS and MD values obtained by microperimetry and Octopus VF did not reveal statistically significant differences between Group 1 and 2 . However, after dividing the examination area into four quadrants, statistically significant intergroup differences in the lower-nasal quadrant were only revealed on microperimetry and not Octopus VF.

The results of kinetic VF test revealed statistically significant differences in the temporal visual field between Group 1 and 3 ( $p=0.000113$ ). No significant differences were noted between Group 1 and 2 . 
Table III. Variables in Group 3

\begin{tabular}{|c|c|c|c|c|c|c|c|}
\hline Group 3 & & $\mathbf{N}$ & Mean & SD & Median & Min & Max \\
\hline \multirow{3}{*}{ Microperimetry [dB] } & MS & 40 & 13.39 & 2.91 & 14.25 & 7.60 & 18.60 \\
\hline & $\mathrm{MD}$ & 40 & -5.10 & 2.65 & -5.40 & -9.00 & 5.70 \\
\hline & MS LN & 40 & 11.61 & 4.65 & 13.63 & 2.70 & 17.70 \\
\hline \multirow{5}{*}{$\begin{array}{l}\text { Contrast sensitivity test } \\
\text { (patch scores) }\end{array}$} & Row A & 40 & 4.05 & 1.34 & 4.15 & 1.00 & 7.00 \\
\hline & Row B & 40 & 4.29 & 1.73 & 4.50 & 0.30 & 7.30 \\
\hline & Row C & 40 & 3.84 & 1.83 & 4.00 & 0 & 7.70 \\
\hline & Row D & 40 & 1.91 & 1.62 & 2.00 & 0 & 5.30 \\
\hline & Row $\mathrm{E}$ & 40 & 0.90 & 1.10 & 0.15 & 0 & 3.70 \\
\hline \multirow{3}{*}{$\begin{array}{l}\text { Static VF } \\
- \text { Octopus [dB] }\end{array}$} & MS & 40 & 19.75 & 4.42 & 20.65 & 9.70 & 27.00 \\
\hline & MD & 40 & 8.26 & 4.92 & 6.50 & 1.30 & 24.40 \\
\hline & MS LN & 40 & 16.09 & 3.58 & 16.20 & 7.00 & 24.90 \\
\hline \multicolumn{2}{|c|}{ Kinetic VF — temporal part [degrees] } & 40 & 48.00 & 31.86 & 60.00 & 0 & 90.00 \\
\hline
\end{tabular}

$\mathrm{N}$ — number; SD — standard deviation; Min — minimum; Max — maximum; dB - decibels; MS — mean sensitivity; MD — mean defect; MS LN — mean sensitivity lower-temporal; VF — visual field

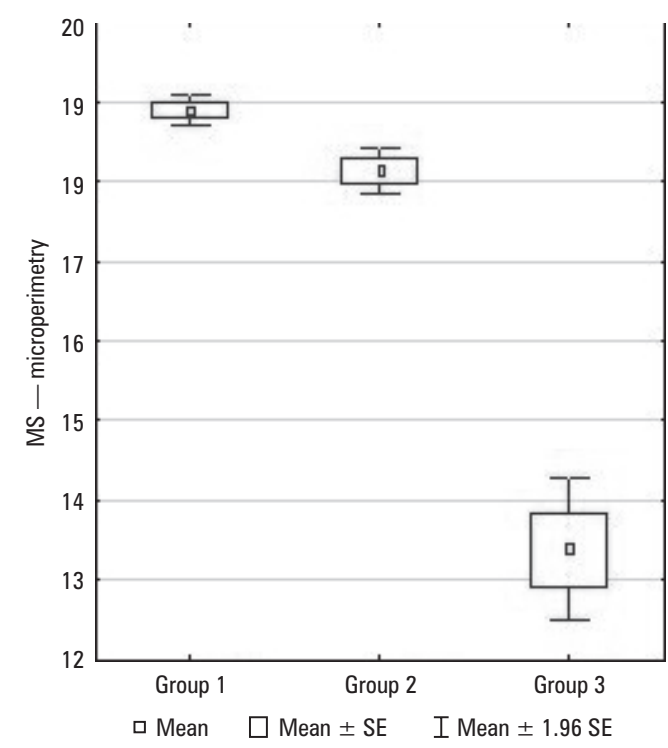

Figure 2. Comparison of MS by microperimetry in three groups. MS - mean sensitivity

Group 3 exhibited a strong correlation between MS LN on microperimetry and temporal visual field $(r=0.914$; Fig. 5). In Group 1 and 2 the relationship between these variables was not that strong.

Receiver operating characteristic curve analysis included MS LN by microperimetry as well as rows D and $\mathrm{E}$ by contrast sensitivity test. A comparison of the ROC curves between Group 1 and 2 revealed the largest area under the curve (AUC) for MS LN (AUC $=0.856$ ). The cut-off value was $\leq 17.9$ with $67.5 \%$ sensitivity and $95 \%$ specificity. In contrast sensitivity test the most informative were row D (AUC $=0.918$ ) with the cut-off value $\leq 4.3$ and $80 \%$ sensitivity, $92.5 \%$ specificity and

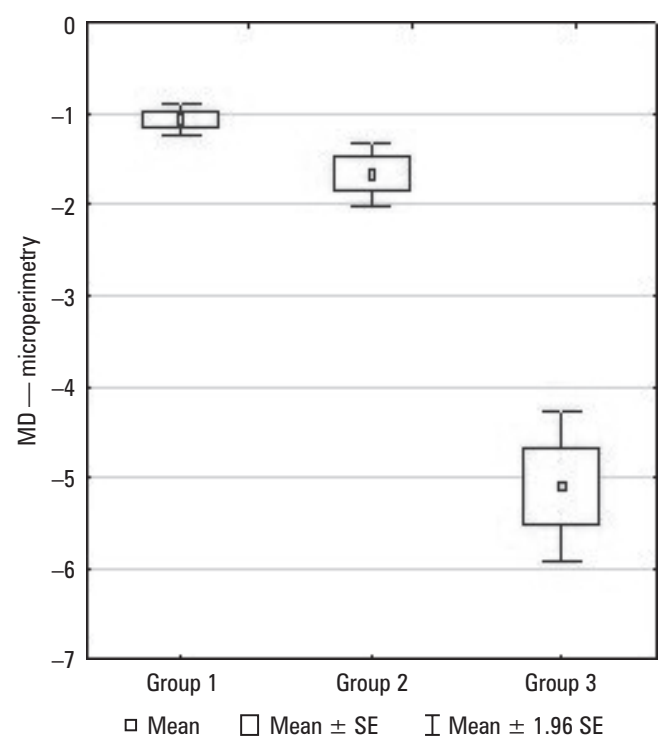

Figure 3. Comparison of $M D$ by microperimetry in three groups. $M D$ - mean defect

row $\mathrm{E}(\mathrm{AUC}=0.856)$ with the cut-off value $\leq 1.7$ and $65 \%$ sensitivity, $100 \%$ specificity (Fig. 6).

\section{Discussion}

Pituitary adenomas can not only lead to different endocrine disorders but tend also to be a serious threat to vision. As a result of the mass effect associated with tumour growth and invasion to adjacent structures, these tumours may cause loss of visual acuity and VF defects [12].

The image passing through the lens and falling on the retina is inverted, both horizontally and verti- 


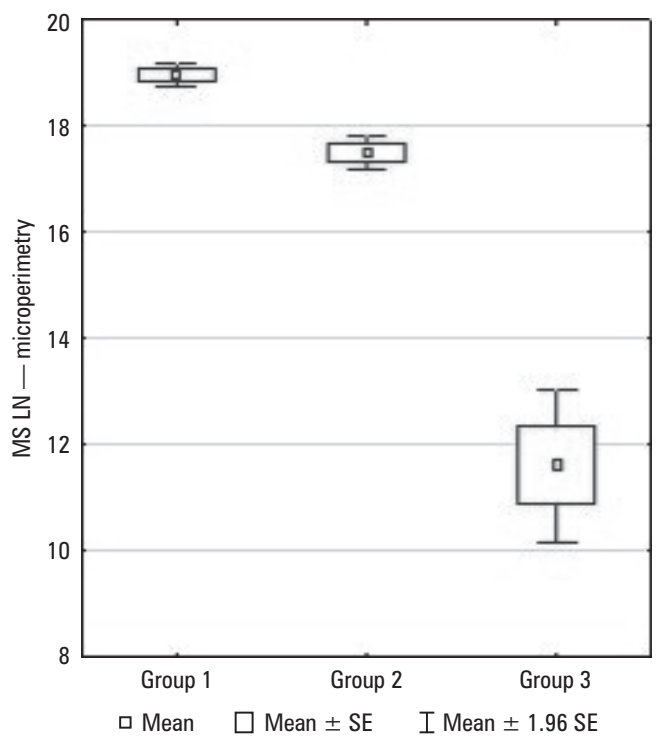

Figure 4. Comparison of MS LN by microperimetry in three groups. MS LN - mean sensitivity lower-temporal; SE - standard error

cally. Therefore, the upper-temporal quadrant of VF is equivalent to the lower-nasal quadrant of the retina. According to Anik, relative or absolute scotomata first develop in the upper-temporal quadrant of VF resulting from a tumour compressing the lower-anterior portion of the optic chiasm. Subsequently, field deficits develop in lower-temporal, lower-nasal, and upper-nasal quadrants [13]. The results of our study showed a similar sequence in microperimetry and static Octopus VF.

Microadenomas or small macroadenomas may disturb blood supply to the optic chiasm and cause VF defects, a phenomenon referred to as intrasellar pressure syndrome [14]. Therefore, we recommend performing microperimetry and contrast sensitivity test not only in people affected by pituitary macroadenoma but also in those with smaller pituitary tumours.

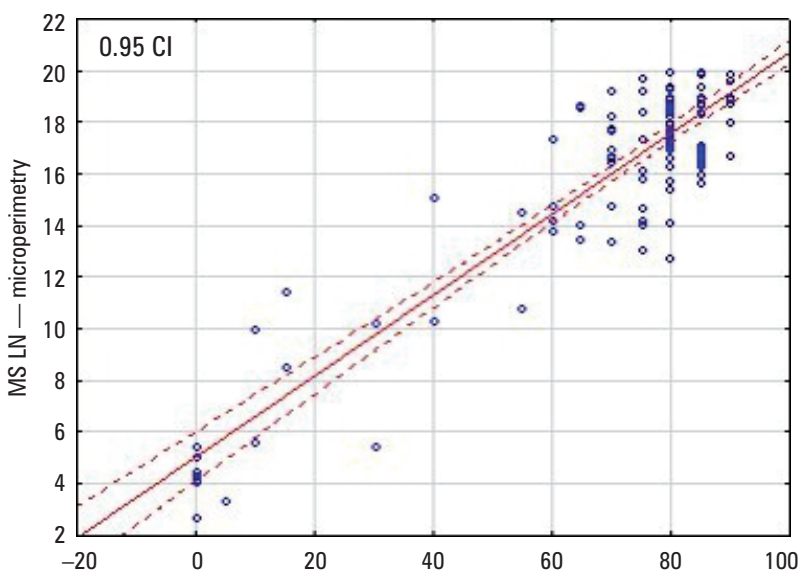

Figure 5. Correlation between MS LN on microperimetry and temporal part of kinetic visual field in Group 3. MS LN - mean sensitivity lower-temporal; CI - confidence interval

There are many limitations to manual kinetic perimetry, e.g. experience of the investigator, diverse response of the patient caused by fatigue, lack of standardisation parameters, and poor repeatability [15]; hence, no accurate assessment of the central VF is possible [16]. Shen et al. concluded that static visual field perimetry was more sensitive than manual Goldmann kinetic perimetry in patients with pituitary adenomas [17], especially in the early stages of the disease [18]. Our results also revealed that the Goldmann perimeter may fail to detect minimal optic chiasm involvements. Hirai et al. examined a group of patients with optic chiasm lesions using a scanning laser ophthalmoscope, a forerunner of microperimetry; they demonstrated that conventional perimetry was not capable of accurate assessment of central retinal sensitivity within $3^{\circ}$ of the fixation point [19]. Microperimetry, on the other hand, offers several advantages; it allows accurate topographic correlation between retinal area details and its light sensitivity [20].
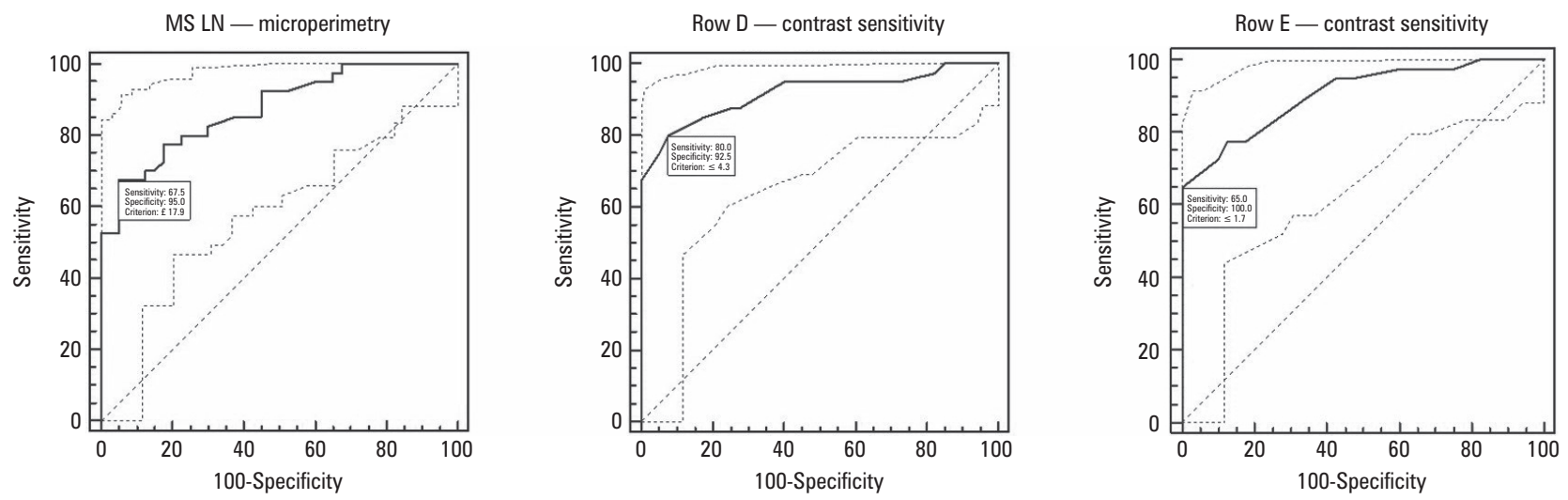

Figure 6. Receiver operating characteristic (ROC) curve analysis of MS LN by microperimetry, rows D and E by contrast sensitivity test. MS LN - mean sensitivity lower-temporal 
Due to considerable interest in microperimetry, the number of publications in this area continues to increase. However, literature on microperimetry and FACT in patients with pituitary adenomas is extremely scarce; in fact, there are only two reports on microperimetry in patients with pituitary adenoma [21]. On one hand this offers a unique opportunity for further studies, and on the other it is difficult to compare the observation of our 40 patients with two case reports.

Furthermore, there are no reports on microperimetry in patients with pituitary macroadenomas but no evidence of obvious chiasmal compression on MRI. To the best of our knowledge, our study is one of the first to describe the application of microperimetry and FACT in a group of patients with pituitary adenomas.

Although microperimetry measures retinal sensitivity at specific points, it does not reveal the MS or MD of each quadrant. In order to accurately assess the disorders caused by chiasmal compression, we divided the examined area into quadrants. In comparison to Octopus VF, microperimetry showed statistically significant differences in MS LN between Group 1 and 2. Patients with pituitary macroadenomas but no signs of chiasmal compression on NMR may be at risk for optic nerve damage, which can be revealed by microperimetry.

Pituitary adenomas may cause bitemporal absolute scotomata while the nasal visual field remains relatively intact. It is therefore crucial that each quadrant should be individually assessed. An analysis of lower-nasal quadrant parameters is of particular importance because it is the first quadrant to become affected by pituitary adenomas. Until now there have been no reports on microperimetry changes in individual retinal quadrants in this group of patients.

In the absence of macular disease, microperimetry facilitates accurate assessment of central retinal sensitivity reduction, secondary to the loss of ganglion cells and their axons [22]. Fundus perimetry complements neuro-ophthalmic examination including visual acuity, colour vision, and conventional VF test [23].

The main limit of microperimetry is the necessity to first obtain high-quality images of the fundus in the infrared in order to superimpose the acquired sensitivity data [24]. In our study we did not encounter any problems obtaining infrared images because the fundus view was not obscured by ocular media opacities in any of the patients.

Microperimeter MP-1 is an interesting diagnostic tool for the detection of early damage to the visual pathway in patients with pituitary adenomas. In subclinical forms of these tumours, microperimetry facilitates detection of abnormalities and allows monitoring of disease progression [19].
Pekel et al. showed that abnormal contrast sensitivity can occur in people with normal visual acuity [25]. Measurement of contrast sensitivity better reflects the functional status of the eye compared to visual acuity examination using high-contrast optotypes [26-28].

Porciatti et al. noted that the assessment of contrast sensitivity could be a valuable tool for early detection and monitoring of visual dysfunction associated with pituitary adenomas [29].

Contrast sensitivity test is particularly useful in the initial stage of pituitary adenomas, including early chiasmal compression, in order to promptly detect damage to optic nerve fibres. This examination should also be performed in the intermediate stages of pituitary adenomas. In patients with large tumours both visual acuity and contrast sensitivity are significantly disturbed. Complete contrast sensitivity loss has also been observed; such patients are unable to perform this test.

Contrast sensitivity can improve following pituitary adenoma resection, especially in patients with smaller contrast sensitivity deficits [30].

The greatest benefit of using ROC curves can be achieved in patients with pituitary macroadenoma detected on NMR but no obvious signs of chiasmal compression (Group 2).

\section{Conclusions}

Microperimetry and contrast sensitivity test are non-invasive diagnostic investigations, adjuncts to NMR, which allow detection of chiasmal compression in the course of pituitary adenoma. They are also important for monitoring tumour progression.

Innovative microperimetry parameters, i.e. MS LN and MD LN, reflect changes in the lower-nasal quadrant of the retina; they are more sensitive for detection of optic chiasm compression compared to MS and MD. In contrast sensitivity test, row $D$ and $E$ values are the most informative. In comparison to kinetic VF and Octopus $\mathrm{VF}$, microperimetry revealed changes in LN quadrant of the retina in Group 2. Microperimetry revealed changes in LN quadrant of the retina in Group 2 undetected on kinetic VF and Octopus VF.

\section{Conflict of interests}

All authors certify that they have no financial interest in the subject matter or materials discussed in this manuscript.

\section{References}

1. Zhan $X$, Wang X, Cheng T. Human pituitary adenoma proteomics: new progresses and perspectives. Front Endocrinol (Lausanne). 2016; 7: 54, doi: 10.3389/fendo.2016.00054, indexed in Pubmed: 27303365. 
2. Brue T, Castinetti F. The risks of overlooking the diagnosis of secreting pituitary adenomas. Orphanet J Rare Dis. 2016; 11(1): 135 , doi: 10.1186/s13023-016-0516-x, indexed in Pubmed: 27716353.

3. Nishioka H. Recent evolution of endoscopic endonasal surgery for treatment of pituitary adenomas. Neurol Med Chir (Tokyo). 2017; 57(4) 151-158, doi: 10.2176/nmc.ra.2016-0276, indexed in Pubmed: 28239067.

4. Malicka JE, Świrska J, Kurowska M, et al. Familial isolated pituitary adenomas (FIPA). Case report of four families and review of literature. Endokrynol Pol. 2017; 68(6): 697-707, doi: 10.5603/EP.a2017.0058, indexed in Pubmed: 29022641.

5. Gilis-Januszewska A, Wilusz M, Pantofliński J, et al. Temozolomide therapy for aggressive pituitary Crooke's cell corticotropinoma causing Cushing's Disease - a case report with literature review. Endokrynol Pol. 2018 69(3): 306-312, doi: 10.5603/EP.a2018.0011, indexed in Pubmed: 29319131

6. Righi A, Agati P, Sisto A, et al. A classification tree approach for pituitary adenomas. Hum Pathol. 2012; 43(10): 1627-1637, doi: 10.1016/j.humpath.2011.12.003, indexed in Pubmed: 22446019.

7. Youssef MM, El-Fayoumi D, Sidky MK, et al. Value of microperimetry in detecting early retinal toxicity of hydroxychloroquine in children with juvenile systemic lupus erythematosus. Ophthalmologica. 2017; 237(3): 180-184, doi: 10.1159/000461595, indexed in Pubmed: 28297699.

8. Hwang J, Seol HoJ, Nam DH, et al. Therapeutic strategy for cavernous sinus-invading non-functioning pituitary adenomas based on the modified Knosp grading system. Brain Tumor Res Treat. 2016; 4(2): 63-69, doi: 10.14791/btrt.2016.4.2.63, indexed in Pubmed: 27867914.

9. Galland F, Vantyghem MC, Cazabat L, et al. Management of nonfunctioning pituitary incidentaloma. Ann Endocrinol (Paris). 2015; 76(3): 191-200, doi: 10.1016/j.ando.2015.04.004, indexed in Pubmed: 26054868

10. Fledelius HC. Temporal visual field defects are associated with monocular inattention in chiasmal pathology. Acta Ophthalmol. 2009; 87(7): 769-775, doi: 10.1111/j.1755-3768.2008.01328.x, indexed in Pubmed: 18976316.

11. Yu YL, Yang YJ, Lin C, et al. Analysis of volumetric response of pituitary adenomas receiving adjuvant CyberKnife stereotactic radiosurgery with the application of an exponential fitting model. Medicine (Baltimore). 2017; 96(4): e4662, doi: 10.1097/MD.0000000000004662, indexed in Pubmed: 28121913.

12. Raverot $\mathrm{G}$, Assié $\mathrm{G}$, Cotton $\mathrm{F}$, et al. Biological and radiological exploration and management of non-functioning pituitary adenoma. Ann Endocrinol (Paris). 2015; 76(3): 201-209, doi: 10.1016/j.ando.2015.04.005, indexed in Pubmed: 26122495.

13. Anik I, Anik Y, Koc K, et al. Evaluation of early visual recovery in pituitary macroadenomas after endoscopic endonasal transphenoidal surgery: Quantitative assessment with diffusion tensor imaging (DTI). Acta Neurochir (Wien). 2011; 153(4): 831-842, doi: 10.1007/s00701-011-0942-4, indexed in Pubmed: 21267606.

14. Hayashi Y, Kita D, Iwato M, et al. Significant improvement of intractable headache after transsphenoidal surgery in patients with pituitary adenomas; preoperative neuroradiological evaluation and intraoperative intrasellar pressure measurement. Pituitary. 2016; 19(2): 175-182, doi: 10.1007/s11102-015-0696-8, indexed in Pubmed: 26659379.

15. Kedar S, Ghate D, Corbett JJ. Visual fields in neuro-ophthalmology. Indian J Ophthalmol. 2011; 59(2): 103-109, doi: 10.4103/0301-4738.77013, indexed in Pubmed: 21350279.

16. Chakravarti T. Assessing Precision of Hodapp-Parrish-Anderson Criteria for Staging Early Glaucomatous Damage in an Ocular Hypertension Cohort: A Retrospective Study. Asia Pac J Ophthalmo
(Phila). 2017; 6(1): 21-27, doi: 10.1097/APO.0000000000000201, indexed in Pubmed: 28161915

17. Shen MQ, Ye W, Zhang YY, et al. [Visual field defects in 169 cases of pituitary adenomas]. Zhonghua Yan Ke Za Zhi. 2009; 45(12): 1074-1079, indexed in Pubmed: 20193428

18. Lilja Y, Gustafsson O, Ljungberg M, et al. Visual pathway impairment by pituitary adenomas: quantitative diagnostics by diffusion tensor imaging. J Neurosurg. 2017; 127(3): 569-579, doi: 10.3171/2016.8.JNS161290, indexed in Pubmed: 27885957.

19. Hirai T, Ito $\mathrm{Y}$, Arai M, et al. Loss of stereopsis with optic chiasmal lesion and stereoscopic tests as a differential test. Ophthalmology. 2002; 109(9): 1692-1702, indexed in Pubmed: 12208719.

20. Acton JH, Greenstein VC. Fundus-driven perimetry (microperimetry) compared to conventional static automated perimetry: similarities, differences, and clinical applications. Can J Ophthalmol. 2013; 48(5): 358-363, doi: 10.1016/j.jcjo.2013.03.021, indexed in Pubmed: 24093180.

21. Romano MR, Angi M, Romano V, et al. Early unilateral macular sensitivity changes in microperimetry in a case of pituitary adenoma. Int Ophthalmol. 2010; 30(6): 709-711, doi: 10.1007/s10792-010-9345-4, indexed in Pubmed: 20177956.

22. Akiba R, Yokouchi H, Mori M, et al. Retinal Morphology and Sensitivity Are Primarily Impaired in Eyes with Neuromyelitis Optica Spectrum Disorder (NMOSD). PLoS One. 2016; 11(12): e0167473, doi: 10.1371/journal.pone.0167473, indexed in Pubmed: 27936154.

23. Mendoza-Santiesteban CE, Hedges III TR, Norcliffe-Kaufmann L, et al. Selective retinal ganglion cell loss in familial dysautonomia. Neurol. 2014; 261(4): 702-709, doi: 10.1007/s00415-014-7258-2, indexed in Pubmed: 24487827.

24. Rohrschneider K, Springer C, Bültmann S, et al. Microperimetry - comparison between the micro perimeter 1 and scanning laser ophthalmoscope - fundus perimetry. Am J Ophthalmol. 2005; 139(1): 125-134 doi: 10.1016/j.ajo.2004.08.060, indexed in Pubmed: 15672526.

25. Pekel G, Alagöz N, Pekel E, et al. Effects of ocular dominance on contrast sensitivity in middle-aged people. ISRN Ophthalmol. 2014; 2014: 903084 , doi: 10.1155/2014/903084, indexed in Pubmed: 24734197.

26. Fatehi N, Nowroozizadeh S, Henry S, et al. Association of structura and functional measures with contrast sensitivity in glaucoma. Am J Ophthalmol. 2017; 178: 129-139, doi: 10.1016/j.ajo.2017.03.019, indexed in Pubmed: 28342719.

27. Liu JL, McAnany JJ, Wilensky JT, et al. M\&S smart system contrast sensitivity measurements compared with standard visual function measurements in primary open-angle glaucoma patients. J Glaucoma. 2017; 26(6): 528-533, doi: 10.1097/IJG.0000000000000659, indexed in Pubmed: 28333894

28. Rodríguez-Vallejo M, Remón L, Monsoriu JA, et al. Designing a new test for contrast sensitivity function measurement with iPad. J Optom. 2015; 8(2): 101-108, doi: 10.1016/j.optom.2014.06.003, indexed in Pubmed: 25890826

29. Porciatti V, Ciavarella P, Ghiggi MR, et al. Losses of hemifield contrast sensitivity in patients with pituitary adenoma and normal visual acuity and visual field. Clin Neurophysiol. 1999; 110(5): 876-886, indexed in Pubmed: 10400201.

30. Slatkeviciene G, Liutkeviciene R, Glebauskiene B, et al. The application of a new maximum color contrast sensitivity test to the early prediction of chiasma damage in cases of pituitary adenoma: the pilot study. Korean J Ophthalmol. 2016; 30(4): 295-301, doi: 10.3341/kjo.2016.30.4.295, indexed in Pubmed: 27478357 